\title{
Metformin ameliorates diabetic nephropathy in a rat model of low-dose streptozotocin-induced diabetes
}

\author{
SIWEI ZHANG* , HUALI XU* ${ }^{*}$ XIAOFENG YU, YI WU and DAYUN SUI \\ Department of Pharmacology, School of Pharmaceutical Sciences, Jilin University, Changchun, Jilin 130021, P.R. China
}

Received March 17, 2015; Accepted April 19, 2016

DOI: $10.3892 / \mathrm{etm} .2017 .4475$

\begin{abstract}
The present study aimed to explore the renoprotective effect of metformin on diabetic nephropathy in type 2 diabetic rats. A rat model of type 2 diabetic nephropathy (T2DN) was successfully induced via a high-fat diet combined with a single low-dose of streptozotocin. Metformin was administered intragastrically for 13 weeks, and fasting blood glucose (FBG), total cholesterol (TC), triglycerides (TG), HDL-c, LDL-c, urinary and serum creatinine levels were subsequently examined at the end of administration. Renal function was determined after the treatment protocol. Expression levels of transforming growth factor (TGF)- $\beta 1$ and connective tissue growth factor (CTGF) were assessed via immunohistochemical analysis. Superoxide dismutase activity, malondialdehyde content and glutathione peroxidase levels were assessed in kidney tissues using commercially available kits. The results of the present study demonstrated that metformin administration significantly decreased the levels of serum blood urea nitrogen, serum creatinine, creatinine clearance, urinary albumin excretion and fasting blood glucose in rats with T2DN. Furthermore, TG, TC and LDL-c levels were significantly decreased following metformin treatment, whereas HDL-c was increased. Metformin treatment significantly increased SOD activity and significantly decreased malondialdehyde levels, as compared with the model group. It was also demonstrated that metformin administration significantly decreased the expression levels of TGF- $\beta 1$ and attenuated the morphological changes associated with T2DN in rats. These data clearly demonstrated the renoprotective effects of metformin against the development and progression of $\mathrm{T} 2 \mathrm{DN}$ in rats. The underlying mechanism of this protective effect may be associated with glycemic control, lipid metabolism, and anti-oxidative and anti-inflammatory functions.
\end{abstract}

Correspondence to: Dr Yi Wu, Department of Pharmacology, School of Pharmaceutical Sciences, Jilin University, 1266 Fujin Road, Changchun, Jilin 130021, P.R. China

E-mail:wuyi@jlu.edu.cn

*Contributed equally

Key words: metformin, captopril, type 2 diabetic nephropathy, rat

\section{Introduction}

Diabetes mellitus is a metabolism-related chronic disease that affects individuals worldwide. Poorly controlled blood glucose levels typically lead to various complications in organs that are rich in microvasculature, including nephropathy (1). Diabetic nephropathy (DN) is a high risk factor for vascular disease, and is common among patients with type 2 diabetes (2). DN is characterized by albuminuria, hyperfiltration and hyperpermeability to macromolecules, proteinuria and end-stage renal failure (3). Although the underlying mechanism of type $2 \mathrm{DN}$ (T2DN) development remains unclear, this multifactorial disease may be associated with factors such as hyperglycemia, hyperlipidemia, oxidative stress and inflammatory cytokines $(4,5)$.

Hyperglycemia causes oxidative stress and increases the generation of reactive oxygen species (ROS) (4). ROS damage the cell membrane, inactivate antioxidant enzymes, alter endogenous antioxidant gene expression and contribute to the pathogenesis of DN (6-8). Furthermore, ROS activate signal transduction cascades which induce the expression of profibrotic factors, including fibronectin, lamin and Collagen IV, which contribute to the accumulation of extracellular matrix (ECM) and inflammatory gene expression, such as IL-6. Furthermore, transforming growth factor (TGF)- $\beta 1$ expression is activated by ROS, in combination with its downstream effector connective tissue growth factor (CTGF), which contributes to tissue fibrosis $(9,10)$ and promotes cell proliferation and ECM synthesis, which is one of the predominant pathological characteristics of DN $(11,12)$.

Metformin, which is an oral hypoglycemic diguanide drug, has been widely used for several decades in the treatment for type 2 diabetes mellitus (T2DM), particularly in patients with obesity (13). Metformin reduces diabetic complications by reducing glucose levels in the body (13); and although the detailed mechanism underlying these benefits is yet to be elucidated, it is generally acknowledged that metformin restores the body's response to insulin. It has been demonstrated that metformin reduces gluconeogenesis in the liver and also inhibits sugar uptake in the intestines (14); whereas another previous study has demonstrated that it may reduce ROS generation (15). Furthermore, metformin has exhibited renal protective effects against a nephrotoxic agent in some previous studies $(16,17)$.

The present study aimed to explore the potential renal protective effect of metformin on the progression of DN in 
high-fat diet (HFD) and STZ-induced diabetic rats, and the underlying mechanism.

\section{Materials and methods}

Regents. Metformin was purchased from Tianjin Pacific Chemical \& Pharmaceutical Co., Ltd., (Tianjin, China). Captopril was purchased from Shanghai Pukang Pharmaceutical Co., Ltd., (Shanghai, China). Detection kits for total cholesterol (TC; A111-1), triglyceride (TG; A110-1), high density lipoprotein-cholesterol (HDL-c; A112-1), low density lipoprotein-cholesterol (LDL-c; S113-1), serum creatinine (Scr), blood urea nitrogen (BUN), superoxide dismutase (SOD; A001-1), malondialdehyde (MDA; A003-1) and glutathione peroxidase (GSH-Px; A005) were purchased from Nanjing Jiancheng Institute of Biotechnology (Nanjing, China).

Animals and experimental design. A total of 80 male Wistar rats, aged 7 weeks and weighing 160-180 g, were obtained from The Experimental Animal Center at Jilin University (Jilin, China). Rats were housed in normal cages under controlled conditions $\left(25^{\circ} \mathrm{C}\right.$ and a $12 \mathrm{~h}$ light/dark cycle) with ad libitum access to a standard pellet diet (SPD) and water. Experiments were performed in accordance with the Guide for the Care and Use of Laboratory Animals of Jilin University, and were approved by the Ethics Committee. Following acclimatization for one week, rats were divided into two groups, and were either fed with SPD or a HFD, consisting of $10 \%$ fat, $20 \%$ sucrose, $10 \%$ protein and $60 \%$ pulverized standard rat pellet, for eight weeks. Following overnight fasting, the rats fed with HFD were intravenously (i.v) injected with a single low-dose of STZ (30 mg $/ \mathrm{kg}$; Sigma-Aldrich, St. Louis, MO, USA), whereas the rats in the control SFD group were treated with a vehicle control buffer. Two weeks following STZ injection, fasting blood glucose (FBG) levels were tested and the rats with high FBG (>11.1 mol/l) were used for the subsequent experiments.

Diabetic rats were randomly divided into three equal groups $(\mathrm{n}=20)$. Experimental groups were as follows: Control, control group; model, diabetic control group; metformin, diabetic group with metformin treatment $(70 \mathrm{mg} / \mathrm{kg})$; and captopril, diabetic group with captopril treatment $(10 \mathrm{mg} / \mathrm{kg})$. Metformin and captopril were administered intragastrically (i.g.) once a day for 13 weeks. Simultaneously, the control and model groups were administered $0.5 \%$ sodium carboxymethyl cellulose (i.g.; Sanpu Chemical Reagent Co., Ltd., Shanghai, China) as procedural control. FBG levels were determined during treatment at $0,3,6,9$ and 13 weeks using a glucometer (Eukare, Eumed Biotechnology Co., Ltd. Taiwan). In the present study, captopril, which is an angiotensin converting enzyme (ACE) inhibitor, was used as a positive control as it has been demonstrated to prevent the progression of chronic kidney disease (18).

Assessment of biochemical parameters. Serum samples were collected to determine HDL-c, LDL-c, TC, TG, SCr and BUN levels. Measurements were performed according to the manufacturer's protocol for each kit. In brief, after 13 weeks of administration the rats were anesthetized by chloral hydrate.
Blood samples were taken from the abdomen artery into non-hepatinised tubes and were allowed to clot for $2 \mathrm{~h}$ at room temperature, and were then centrifuged at $1,100 \mathrm{xg}$ for $15 \mathrm{~min}$.

Assessment of urine parameters. The urinary creatinine and creatinine in plasma were measured using an AU5800 automatic analyzer (Beckman Coulter, Inc., CA, USA). Creatinine clearance $(\mathrm{CCr})$ and 24-h urinary albumin excretion rate (UAER) were calculated according to the following formulae: UAER=urinary albumin $(\mu \mathrm{g} / \mathrm{ml}) \times 24-\mathrm{h}$ urine volume $(\mathrm{ml})$; and $\mathrm{CCr}=$ urinary creatinine $(\mathrm{UCr})(\mathrm{mg} / \mathrm{ml}) \mathrm{x}$ urine volume $(\mathrm{ml} / \mathrm{kg}) /$ creatinine in plasma $(\mathrm{mg} / \mathrm{ml})(19)$.

Determination of SOD, MDA and GSH-Px levels. One small section $(200 \mathrm{mg}$ ) of right kidney was harvested from the rats and precisely weighed. Subsequently, saline was added according to the tissue weight: Saline volume=1:9 (w/v). Following homogenization at $4^{\circ} \mathrm{C}$ by a DY89-I electric homogenate (Ningbo Scientz Biotechnology Co., Ltd., Ningbo, China), the homogenates were centrifuged at 1,100xg for $15 \mathrm{~min}$ at room temperature. SOD activity, MDA content and GSH-Px levels were measured using commercially available kits, according to manufacturer's protocol.

Histopathology and electron microscopy examination. Kidney samples were fixed in $10 \%$ formalin, paraffin-embedded, cut into 5-micron sections and stained with hematoxylin and eosin (Sanpu Chemical Reagent Co., Ltd.). Slides were examined under an Eclipse 80i light microscope (magnification, $\mathrm{x} 400$; Nikon, Tokyo, Japan) by a pathologist who was blind to the experimental profiles. For electron microscopy, kidney tissues were cut into small cubes $(2 \mathrm{~mm} / \mathrm{side})$, fixed in $4 \%$ glutaraldehyde and examined by independent pathologists with a JEM-1200EX transmission electron microscope (Japan Electronic Co., Ltd.) who were blinded to the protocol of the present study.

Immunohistochemical analysis. Expression levels of TGF- $\beta 1$ and CTGF in the kidney sections were analyzed via immunochemical staining. Polyclonal anti-rabbit TGF- $\beta 1$ antibody (1:200; bs-7443R) and monoclonal anti-rabbit CTGF antibody (1:500; bs-0743R) were purchased from Boaosen (Wuhan, China). Brown/yellow granular deposits in the cells or matrix were interpreted as positive. Semi-quantitative evaluations of the images were carried out using Image Pro Plus 6.0 (Media Cybernetics, Inc., Rockville, MD, USA).

Statistical analysis. Data are presented as the mean \pm standard error of the mean. Statistical significance was determined by one-way analysis of variance, followed by Dunnett's test. $\mathrm{P}<0.05$ was considered to indicate a statistically significant difference.

\section{Results}

Metformin decreases FBG levels in T2DN rats. Following STZ injection, the rats in the model group exhibited the typical symptoms of diabetes, with FBG levels $>11.1 \mathrm{mmol} / 1$. As shown in Fig. 1, the FBG levels in the model group significantly increased as the experimental period increased. From the third 
Table I. Effects of metformin on blood lipids in rats with type 2 diabetes nephropathy.

\begin{tabular}{lllll}
\hline Group & $\begin{array}{c}\text { LDL-c } \\
(\mathrm{mmol} / \mathrm{l})\end{array}$ & $\begin{array}{c}\text { HDL-c } \\
(\mathrm{mmol} / \mathrm{l})\end{array}$ & $\begin{array}{c}\text { TG } \\
(\mathrm{mmol} / \mathrm{l})\end{array}$ \\
\hline Control & $0.54 \pm 0.07$ & $0.65 \pm 0.07$ & $0.27 \pm 0.04$ & $1.60 \pm 0.10$ \\
Model & $1.02 \pm 0.09^{\mathrm{a}}$ & $0.42 \pm 0.04^{\mathrm{a}}$ & $0.42 \pm 0.04^{\mathrm{b}}$ & $2.12 \pm 0.15^{\mathrm{b}}$ \\
Metformin & $0.62 \pm 011^{\mathrm{c}}$ & $0.58 \pm 0.05^{\mathrm{d}}$ & $0.27 \pm 0.03^{\mathrm{c}}$ & $1.66 \pm 0.13^{\mathrm{c}}$ \\
Captopril & $0.80 \pm 0.10$ & $0.55 \pm 0.05$ & $0.39 \pm 0.07$ & $1.68 \pm 0.23$ \\
\hline
\end{tabular}

${ }^{a} \mathrm{P}<0.01$ and ${ }^{b} \mathrm{P}<0.05$, vs. the normal group; ${ }^{\mathrm{c}} \mathrm{P}<0.05$ and ${ }^{\mathrm{d}} \mathrm{P}<0.01$, vs. the model group.

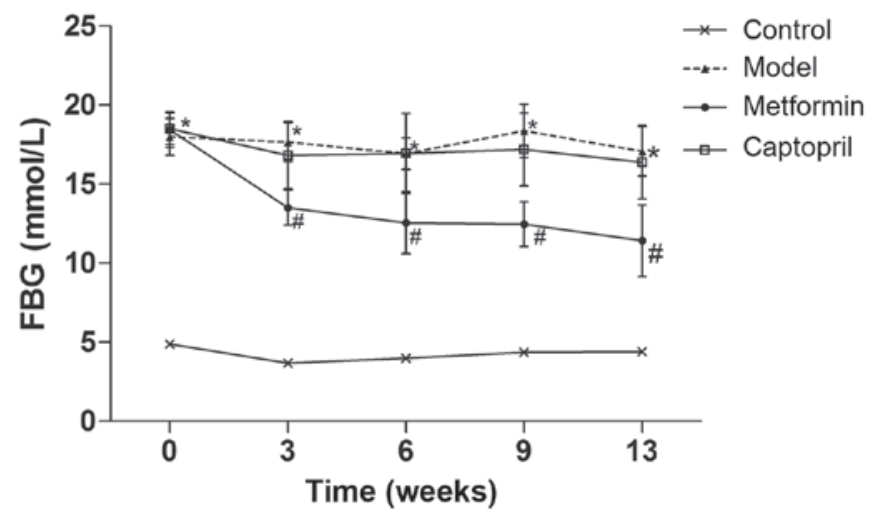

Figure 1. Effects of metformin on fasted blood glucose (FBG) in rats with type 2 diabetes nephropathy. Time course monitoring of FBG levels with daily metformin and captopril for 13 weeks are shown. Data are presented as the mean \pm standard error of the mean. ${ }^{*} \mathrm{P}<0.05$ vs. the normal group; ${ }^{\prime} \mathrm{P}<0.05$ vs. the model group.

A

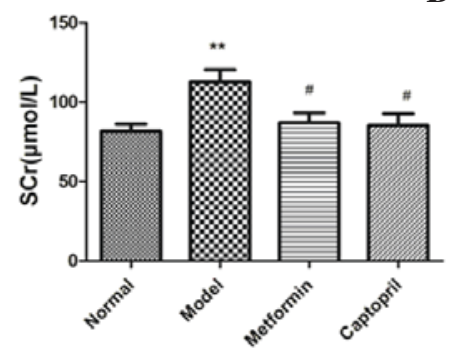

B

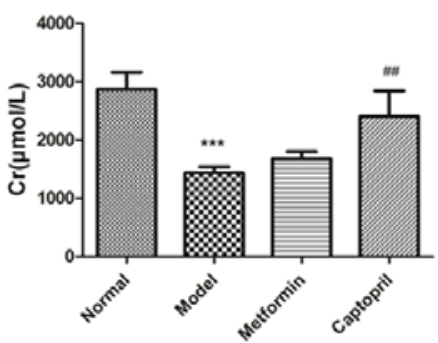

C

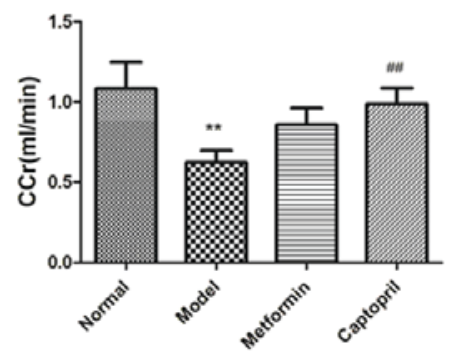

D

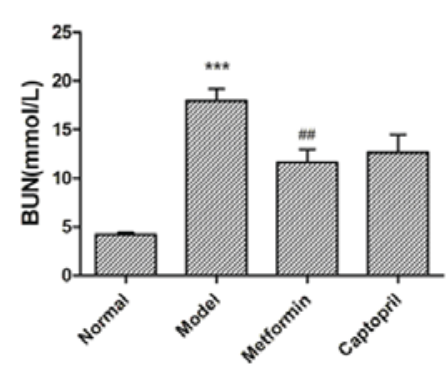

$\mathbf{E}$

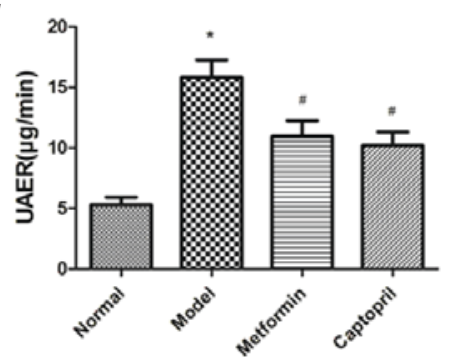

Figure 2. Effects of metformin on kidney function in rats with type 2 diabetes nephropathy. (A) Serum creatinine (SCr), (B) urine creatinine (UCr), (C) creatinine clearance (CCr), (D) blood urea nitrogen (BUN) and (E) 24-h urinary albumin excretion rate (UAER) were assessed. Data are presented as the mean \pm standard error of the mean. ${ }^{*} \mathrm{P}<0.05,{ }^{* *} \mathrm{P}<0.01$ and ${ }^{* * *} \mathrm{P}<0.001$, vs. the normal group; ${ }^{\#} \mathrm{P}<0.05$ and ${ }^{\# \# /} \mathrm{P}<0.01$, vs. the model group.

week onwards, the metformin group exhibited significantly decreased FBG levels $(\mathrm{P}<0.05)$, whereas the captopril group exhibited no significant differences in FBG. These results suggest that metformin decreases FBG in T2DN rats. 
A

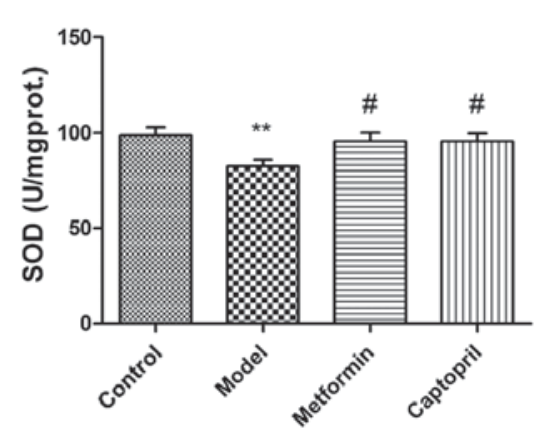

B

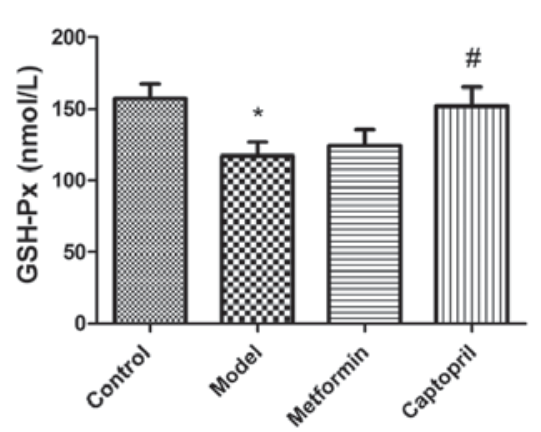

C

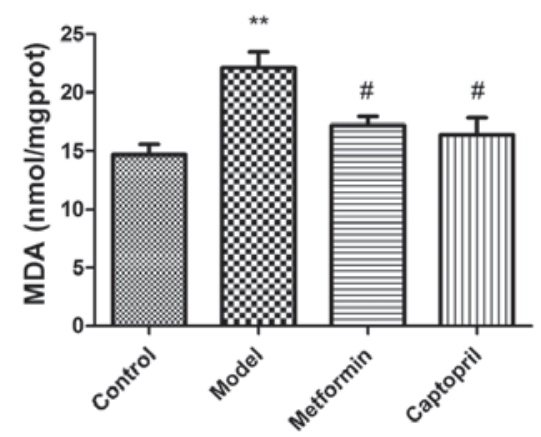

Figure 3. Effects of metformin on (A) superoxide dismutase (SOD), (B) glutathione peroxidase (GSH-Px) and (C) malondialdehyde (MDA) oxidative parameters in the kidney tissues of rats with type 2 diabetes nephropathy. Data are presented as the mean \pm standard error of the mean. ${ }^{*} \mathrm{P}<0.05$ and ${ }^{* *} \mathrm{P}<0.01, \mathrm{vs}$. the normal group; ${ }^{\#} \mathrm{P}<0.05$, vs. the model group.
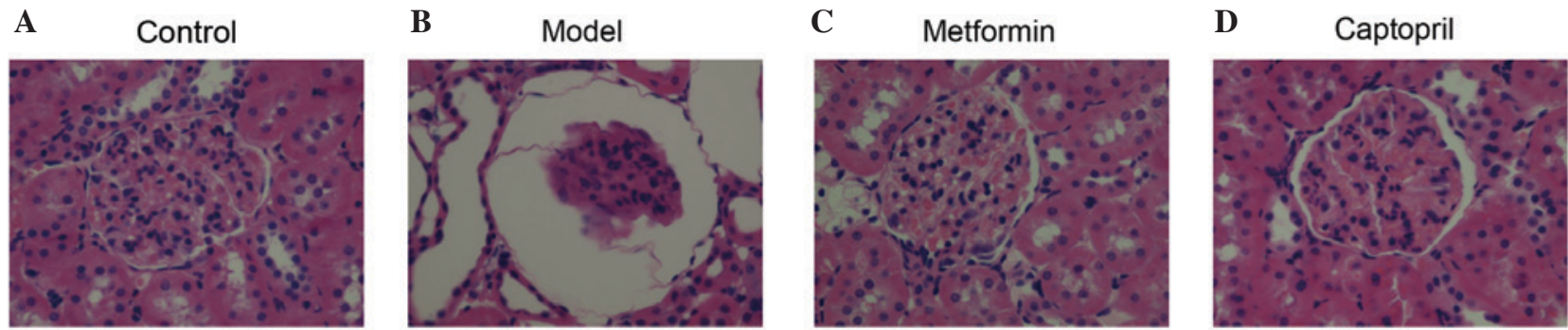

Figure 4. Effect of metformin on the histological morphology of kidneys harvested from rats with type 2 diabetes nephropathy, as detected by hematoxylin and eosin staining. (A) Normal rats, (B) Model rats, (C) Metformin-treated rats and (D) Captopril-treated rats (magnification, x400).

Metformin protects the kidney function in T2DN rats. Following the induction of hyperglycemia via STZ, significant increases in $\mathrm{SCr}(\mathrm{P}<0.01)$, BUN $(\mathrm{P}<0.001)$ and UAER levels were detected, accompanied by significant decreases in $\mathrm{UCr}(\mathrm{P}<0.001)$ and $\mathrm{CCr}(\mathrm{P}<0.01)$, which indicated a significant alternation in the kidney function of model rats, as compared with the control rats (Figs. 2A-E). Metformin treatment significantly reduced $\mathrm{SCr}(\mathrm{P}<0.05)$ and $\mathrm{BUN}(\mathrm{P}<0.01)$ and UAER $(\mathrm{P}<0.05)$ levels in diabetic rats, as compared with the model group. Metformin did not induce any significant alterations in $\mathrm{UCr}$ and $\mathrm{CCr}$ levels; whereas $\mathrm{UCr}$ and $\mathrm{CCr}$ levels were significantly increased following captopril treatment, as compared with the model group $(\mathrm{P}<0.01)$. These results suggest that metformin protects rental function in $\mathrm{T} 2 \mathrm{DN}$ rats.

Metformin decreases blood lipids in T2DN rats. Diabetic dyslipidemia was successfully established in rats, as indicated by increased LDL-c $(\mathrm{P}<0.01), \mathrm{TG}$ and TC (both $\mathrm{P}<0.05)$ levels, and decreased HDL-c $(\mathrm{P}<0.01)$ levels. Metformin treatment significantly counteracted the above mentioned alterations in diabetic rats; serum LDL-c, TG and TC were reduced $(\mathrm{P}<0.05)$ and HDL-c was increased $(\mathrm{P}<0.01)$. Captopril had no significant effect on hyperlipidemia (Table I). These results suggest that metformin decreases blood lipids in T2DN rats.

Metformin decreases oxidative stress in T2DN rats. Diabetic rats exhibited significantly decreased activities of SOD $(\mathrm{P}<0.01)$ and GSH-Px $(\mathrm{P}<0.05)$ (Figs. 3A and $\mathrm{B})$, which was accompanied by a significant increase in MDA levels $(\mathrm{P}<0.01)$ (Fig. 3C) in kidney tissue, as compared with the control rats.
Metformin treatment significantly increased the activity of SOD $(\mathrm{P}<0.05)$ and decreased the level of MDA in diabetic rats, as compared with the model group. However, the activity of GSH-Px was only increased by captopril administration $(\mathrm{P}<0.05)$, as compared with the model group. These results suggest that metformin inhibits oxidative stress in T2DN rats.

Metformin protects histopathologcial changes of kidney in $T 2 D N$ rats. Fig. 4 shows the morphology of the glomerulus, as detected by hematoxylin-eosin staining. As compared with the control group (Fig. 4A), model rats exhibited reduced glomerular tuft, increased Bowman's spaces, vacuolar degeneration, and dilated renal capsule and kidney tubules (Fig. 4B). Metformin and captopril markedly improved the renal lesions in diabetic rats (Figs. $4 \mathrm{C}$ and $\mathrm{D}$, respectively).

Representative images of the kidney cortices acquired by transmission electron microscope are presented in Fig. 5. Kidney cortices samples from the control group exhibited normal structure of three layer of the filtration barrier, with normal glomerular basement membrane (GBM) thickness and equally distributed podocyte foot processes that form foot-like structures (Fig. 5A-a). In the model rats, the podocytes processes merged together in large areas, and the filtration barrier was blurry and no longer clearly distinguishable (Fig. 5A-b ). Furthermore, significant segmental thickening of the GBM was detected $(\mathrm{P}<0.0001)$, as compared with the control group (Fig. 5B). Treatment with metformin and captopril ameliorated these ultrastructural changes in the kidneys of diabetic rats (Figs. 5A-c and 5A-d). Notably, GBM thickness was significantly decreased in the metformin- and 

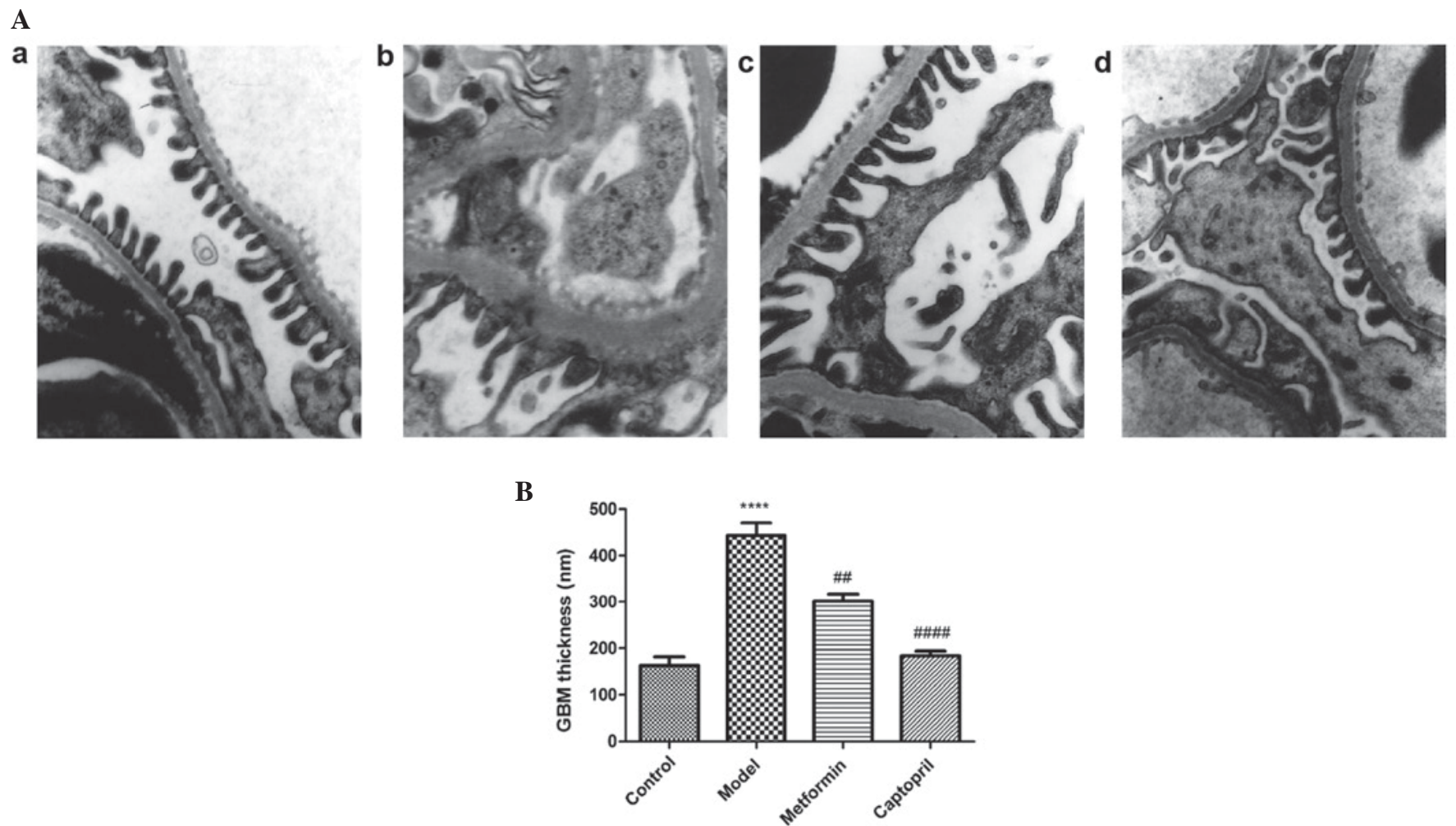

Figure 5. Representative transmission electron microscopy images of kidney cortices (A) in (a) normal, (b) model, (c) metformin-treated and (d) captopril-treated rats (magnification, x15,000). (B) Quantitative analysis of glomerular basement membrane (GBM) thickness. Data are presented as the mean \pm standard error of the mean. ${ }^{* * * *} \mathrm{P}<0.0001$, vs. the normal group; ${ }^{\# \#} \mathrm{P}<0.01$ and ${ }^{\# \# \# \#} \mathrm{P}<0.0001$, vs. the model group.
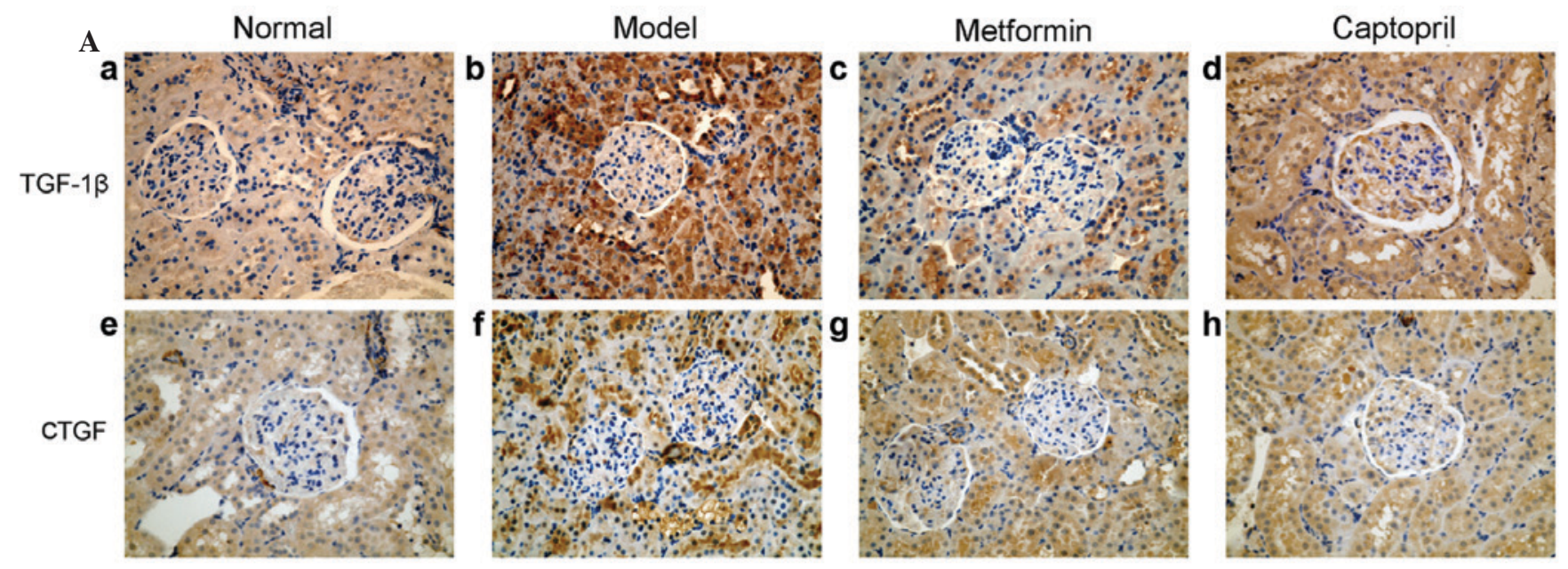

B
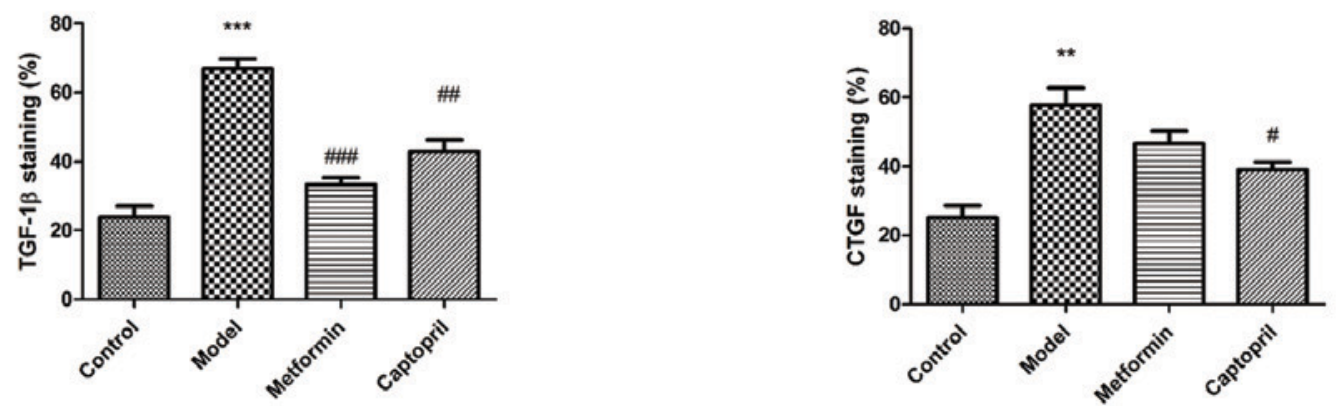

Figure 6. Effects of metformin on TGF- $\beta 1$ and CTGF expression levels in the kidney tissue of rats with type 2 diabetes nephropathy. (A) Kidney tissues from (a and e) normal rats, (b and f) diabetic rats, (c and $\mathrm{g}$ ). metformin-treated diabetic rats and ( $\mathrm{d}$ and $\mathrm{h}$ ) captopril-treated diabetic rats were stained with anti-TGF- $\beta 1$ and anti-CTGF antibodies (magnification, $x 400$ ). (B) Semi-quantitative analysis of TGF- $\beta 1$ and CTGF immunostaining in the glomeruli and renal tubules. Data are presented as the mean \pm standard error of the mean. ${ }^{* *} \mathrm{P}<0.01$ and ${ }^{* * * *} \mathrm{P}<0.001$, vs. the normal group; ${ }^{\#} \mathrm{P}<0.05$, ${ }^{\# \#} \mathrm{P}<0.01$ and ${ }^{\# \# \#} \mathrm{P}<0.001$, vs. the model group. TGF, transforming growth factor; CTGF, connective tissue growth factor. 
captopril-treated groups, as compared with the model group $(\mathrm{P}<0.01$ and $\mathrm{P}<0.0001$, respectively). These results suggest that metformin protects against glomerular damage in the kidneys of T2DN rats.

Metformin decreases TGF- $\beta 1$ and CTGF expression levels in $T 2 D N$ rats. The effect of metformin treatment on the expression levels of TGF- $\beta 1$ and CTGF were examined by immunohistochemistry. As shown in Fig. 6, positive staining of TGF- $\beta 1$ and CTGF in the proximal tubule and glomerular mesangial area were significantly enhanced in the model group, as compared with the control group. As compared with the model group, TGF- $\beta 1$ expression levels were significantly reduced by metformin and captorpil treatment $(\mathrm{P}<0.001$ and $\mathrm{P}<0.01$, respectively). Only captopril administration was able to significantly reduce the expression levels of CTGF, as compared with the model group $(\mathrm{P}<0.05)$.

Semi-quantitative analysis of the positively stained areas demonstrated that TGF- $\beta 1$ and CTGF expression levels in the DN rats were increased, whereas metformin treatment was able to significantly reduce the expression of TGF- $\beta 1$ $(\mathrm{P}<0.001$; Fig. 6B). These results suggest that metformin decreases TGF- $\beta 1$ and CTGF expression levels in the kidneys of $\mathrm{T} 2 \mathrm{DN}$ rats.

\section{Discussion}

In the present study, the renoprotective effects of metformin against T2DN were demonstrated in a high-fat diet, low-dose STZ-induced rat model of T2DN. These results demonstrated that merformin can markedly improve the renal lesions (Fig. 4) and ameliorate the GBM thickness of the kidney (Fig. 5) in diabetic rats. At the same time, merformin can decrease the FBG levels, reduce TG and TC levels, decrease the TGF- $\beta 1$ expression and increase SOD activity. Therefore, treatment with metformin indicated the importance of glycemic control, blood lipid control, anti-oxidative and anti-inflammatory effects in the treatment of T2DN.

STZ is widely used in studies investigating DM, as it specifically targets $\beta$-cells and reduces blood insulin levels, leading to hyperglycemia and mimicking DM pathology (20-22). Dosages of STZ required in DM models remain controversial. In a pervious mouse model, high-dose STZ induced direct nephrotoxicity, making it unable to distinguish between the direct toxic effect of STZ and the lesions that resulted from STZ-induced hyperglycemia $(20,23,24)$. In the present study, insulin resistance was induced in a rat model via a high-fat diet $(25,26)$, followed by a single low dose of STZ $(30 \mathrm{mg} / \mathrm{kg})$ to induce hyperglycemia for 2 weeks. Elevated glucose levels after 13 weeks successfully induced renal lesions that were similarly present in human patients with $\mathrm{DN}$, hyperglycemia, hyperlipidemia, oxidative stress and renal damage (20). According to previous diagnostic criteria of DN outlined by Mogensen et al (27), the present study evaluated rats in DN stages 3-4.

In the present STZ-induced DN model, kidney damage was assessed via kidney function parameters, including elevated $\mathrm{SCr}, \mathrm{UCr}, \mathrm{BUN}$ and UAER, and declined $\mathrm{CCr}$ levels. Presence of albumin in the urine is a primary indicator of kidney damage in the early onset of DN, and the UAER is typically measured in the clinic to assess renal lesions in patients with DN $(28,29)$. Creatinine is a breakdown product of creatine phosphate in muscle and its clearance rate from blood to urine $(\mathrm{CCr})$ correlates with glomerular filtration rate (30); therefore, $\mathrm{CCr}$ can be used as an indicator for kidney function. Kidney damage, as indicated by reduced UAER, was detected 13 weeks after the initiation of STZ-induced hyperglycemia. In addition, SCr, $\mathrm{UCr}$ and BUN kidney function parameters were significantly increased in the model group, as compared with the control group. Treatment with metformin alone ameliorated the majority of these kidney dysfunctions, indicating the protective effects of metformin administration in rats with T2DN. In the model group, morphological and ultrastructural analysis indicated severe damage to the kidney tissue, including reduced glomeruli, dilation of the renal capsule and kidney tubules, and GBM thickening. Treatment with metformin successfully counteracted these morphological alterations.

Hyperglycemia is considered to be the dominant pathological characteristic of DM, as it causes the majority of the symptoms associated with DM. Chronic hyperglycemia results in the accumulation of advanced glycation end products (AGEs) in the body, which further increase the risk for vascular complications in patients with DM (31). A previous study demonstrated that AGEs are always present in diabetic individuals with kidney dysfunction, leading to the overproduction of ROS (32). ROS produced by hyperglycemia are capable of damaging cell membranes and inactivating endogenous antioxidants, lipid and carbohydrate $(33,34)$, and are considered to be a predominant cause of diabetic-related complications $(35,36)$. Endogenous antioxidant molecules, such as SOD and GSH-Px, counteract ROS-mediated renal injury (37); however, they are severely decreased in patients with T2DN, indicating oxidative stress $(37,38)$. As hyperglycemia has a dominant role at the beginning of the ROS-mediated pathway, strict glucose modulation remains the major therapeutic strategy, as this aids the amelioration of oxidative stress (39-41). In the present study, elevated FBG levels were significantly reduced by the well-characterized hypoglycemic agent, metformin; whereas the ACE inhibitor, captopril, induced no effect on FBG. These results indicated that glycemic control is important for the metformin-mediated renoprotective effect. Furthermore, the levels of SOD, GSH-Px and MDA in kidney homogenates were determined. In the model group, decreased levels of the SOD and GSH-Px antioxidants were accompanied by an increase in MDA levels. Treatment with metformin or captopril increased SOD levels and reduced MDA levels. These results suggested that treatment with metformin or captopril exerts a renoprotective effect via increasing SOD and reducing MDA.

Hyperlipidemia is a common symptom of DM (42-45). Persistent filtration of lipids and lipid proteins contributes to chronic and progressive renal injury (46), albuminuria and glomerulosclerosis $(46,47)$. Hyperlipidemia is considered as a risk factor for DN as it may exacerbate glomerular injury through the activation of various signaling pathways (48). The lipid profile of patients with DN is as follows: High LDL-c, TC and TG, and low HDL-c $(45,49)$. This lipid profile has been termed 'diabetic dyslipidemia', and is predominantly observed in patients with T2DM (49). Notably, it has been demonstrated that these lipid abnormalities are associated with worsening kidney function and urinary albumin excretion (49). In the 
present study, the model group exhibited increased TC, TG and LDL-c, and decreased HDL-c. Treatment with metformin, which is a hypoglycemic drug, significantly attenuated these pathological alternations in blood lipid concentrations; whereas captopril exhibited no significant effect. This result implied that metformin may help ease DN symptoms by modulating lipid metabolism and dyslipidemia.

There is a growing body of evidence suggesting the involvement of inflammatory cytokines, including TGF- $\beta 1$, CTGF and IL-6, in the pathogenesis of DN $(50,51)$. Oxidative stress activates TGF- $\beta 1$ and IL- 6 by modulating multiple pathways, and these cytokines contribute to glomerular mesangial expansion and tubulointerstitial fibrosis $(39,40,50)$. CTGF expression is associated with oxidative stress and has been established as the downstream effector of TGF- $\beta 1$ (52). Together, CTGF and TGF- $\beta 1$ contribute to tissue fibrosis $(9,10)$, promoting cell proliferation and ECM synthesis (11). Furthermore, it has previously been demonstrated that serum IL-6 levels were significantly elevated in patients with T2DN, as compared with hyperglycemic patients without nephropathy $(53,54)$. Elevation of IL-6 inflammatory cytokine levels in patients with DM and nephropathy indicates that T2DN is a low-inflammatory disease $(51,55)$. Therefore, the use of agents with anti-inflammatory effects may serve as an attractive strategy for the treatment of renal dysfunction in patients with T2DM (40). In the present study, uncontrolled nephropathy was detected in the model group, which was characterized by significantly elevated expression levels of TGF- $\beta 1$ and CTGF in the kidney. The results of the present study demonstrated that metformin was capable of counteracting the inflammation effect of DN and prevent chronic DN pathogenesis. Therefore, the anti-inflammatory effect induced by metformin is another important mechanism by which it protected rats from the development of chronic T2DN in the present study.

In conclusion, the present study demonstrated the renoprotective effects of metformin against T2DN in a high-fat diet, low-dose STZ-induced rat model of T2DN. The successful induction of T2DN in the rats was demonstrated by severe renal dysfunction, hyperglycemia, dyslipidemia, a reduction in antioxidants and increased expression levels of inflammatory cytokines. Metformin treatment significantly attenuated the pathological characteristics of T2DN, by reducing blood glucose, protecting renal functions and retaining normal morphology. The mechanism of this effect may be associated with glycemic control, lipid metabolism, and anti-oxidative and anti-inflammatory functions; however, further investigation is required to fully elucidate the underlying mechanism.

\section{References}

1. Kashihara N, Haruna Y, Kondeti VK and Kanwar YS: Oxidative stress in diabetic nephropathy. Curr Med Chem 17: 4256-4269, 2010.

2. Yokoyama H, Araki S, Kawai K, Hirao K, Oishi M, Sugimoto K, Sone H, Maegawa $\mathrm{H}$ and Kashiwagi A; Japan Diabetes Clinical Data Management Study Group: Pioglitazone treatment and cardiovascular event and death in subjects with type 2 diabetes without established cardiovascular disease (JDDM 36). Diabetes Res Clin Pract 109: 485-492, 2015.

3. Ritz E: Diabetic nephropathy. Saudi J Kidney Dis Transpl 17: 481-490, 2006.

4. Zou J, Yu X, Qu S, Li X, Jin Y and Sui D: Protective effect of total flavonoids extracted from the leaves of Murraya paniculata (L.) Jack on diabetic nephropathy in rats. Food Chem Toxicol 64: 231-237, 2014
5. Gohda T, Mima A, Moon JY and Kanasaki K: Combat diabetic nephropathy: From pathogenesis to treatment. J Diabetes Res 2014: 207140, 2014.

6. Pan HZ, Zhang L, Guo MY, Sui H, Li H, Wu WH, Qu NQ, Liang MH and Chang D: The oxidative stress status in diabetes mellitus and diabetic nephropathy. Acta Diabetol 47 (Suppl 1): S71-S76, 2010.

7. Swaminathan S and Shah SV: Novel approaches targeted toward oxidative stress for the treatment of chronic kidney disease. Curr Opin Nephrol Hypertens 17: 143-148, 2008.

8. Tabak O, Gelisgen R, Erman H, Erdenen F, Muderrisoglu C, Aral $\mathrm{H}$ and Uzun $\mathrm{H}$ : Oxidative lipid, protein and DNA damage as oxidative stress markers in vascular complications of diabetes mellitus. Clin Invest Med 34: E163-E171, 2011.

9. Ho C, Lee PH, Hsu YC, Wang FS, Huang YT and Lin CL: Sustained $W n t / \beta$-catenin signaling rescues high glucose induction of transforming growth factor- $\beta 1$-mediated renal fibrosis. Am J Med Sci 344: 374-382, 2012.

10. Zorena K, Malinowska E, Raczyńska D, Myśliwiec M and Raczyńska K: Serum concentrations of transforming growth factor-Beta 1 in predicting the occurrence of diabetic retinopathy in juvenile patients with type 1 diabetes mellitus. J Diabetes Res 2013: 614908, 2013.

11. Chen MM, Lam A, Abraham JA, Schreiner GF and Joly AH: CTGF expression is induced by TGF-beta in cardiac fibroblasts and cardiac myocytes: A potential role in heart fibrosis. J Mol Cell Cardiol 32: 1805-1819, 2000.

12. Li X, Cui X, Sun X, Li X, Zhu Q and Li W: Mangiferin prevents diabetic nephropathy progression in streptozotocin-induced diabetic rats. Phytother Res 24: 893-899, 2010.

13. Nasri H and Rafieian-Kopaei M: Metformin: Current knowledge. J Res Med Sci 19: 658-664, 2014.

14. Kirpichnikov D, McFarlane SI and Sowers JR: Metformin: An update. Ann Intern Med 137: 25-33, 2002.

15. Grossmann ME, Yang DQ, Guo Z, Potter DA and Cleary MP: Metformin Treatment for the Prevention and/or Treatment of Breast/Mammary Tumorigenesis. Curr Pharmacol Rep 1: 312-323, 2015.

16. Rafieian-Kopaei M and Nasri H: Ginger and diabetic nephropathy. J Renal Inj Prev 2: 9-10, 2013.

17. Baradaran A: Lipoprotein(a), type 2 diabetes and nephropathy; the mystery continues. J Nephropathol 1: 126-129, 2012.

18. Kim CS, Sohn EJ, Kim YS, Jung DH, Jang DS, Lee YM, Kim DH and Kim JS: Effects of KIOM-79 on hyperglycemia and diabetic nephropathy in type 2 diabetic Goto-Kakizaki rats. J Ethnopharmacol 111: 240-247, 2007.

19. Kong LL, Wu H, Cui WP, Zhou WH, Luo P, Sun J, Yuan H and Miao LN: Advances in murine models of diabetic nephropathy. J Diabetes Res 2013: 797548, 2013.

20. Dupuis F, Atkinson J, Limiñana P and Chillon JM: Captopril improves cerebrovascular structure and function in old hypertensive rats. Br J Pharmacol 144: 349-356, 2005.

21. Szkudelski T: The mechanism of alloxan and streptozotocin action in B cells of the rat pancreas. Physiol Res 50: 537-546, 2001.

22. Lenzen S: The mechanisms of alloxan-and streptozotocin-induced diabetes. Diabetologia 51: 216-226, 2008.

23. Hall-Craggs M, Brenner DE, Vigorito RD and Sutherland JC: Acute renal failure and renal tubular squamous metaplasia following treatment with streptozotocin. Hum Pathol 13: 597-601, 1982.

24. Tay YC, Wang Y, Kairaitis L, Rangan GK, Zhang C and Harris DC: Can murine diabetic nephropathy be separated from superimposed acute renal failure? Kidney Int 68: 391-398, 2005.

25. Jiang $\mathrm{X}, \mathrm{Ma} \mathrm{H}$, Wang $\mathrm{Y}$ and Liu Y: Early life factors and type 2 diabetes mellitus. J Diabetes Res 2013: 485082, 2013.

26. Lee KM, Yang SJ, Kim YD, Choi YD, Nam JH, Choi CS, Choi HS and Park CS: Disruption of the cereblon gene enhances hepatic AMPK activity and prevents high-fat diet-induced obesity and insulin resistance in mice. Diabetes 62: 1855-1864, 2013.

27. Mogensen CE, Christensen CK and Vittinghus E: The stages in diabetic renal disease. With emphasis on the stage of incipient diabetic nephropathy. Diabetes 32 (Suppl 2): S64-S78, 1983.

28. Viberti $\mathrm{G}$ and Wheeldon NM; MicroAlbuminuria Reduction With VALsartan (MARVAL) Study Investigators: Microalbuminuria reduction with valsartan in patients with type 2 diabetes mellitus: A blood pressure-independent effect. Circulation 106: 672-678, 2002.

29. Wang XL, Lu JM, Pan CY and Tian H: A study comparing the prevalence of urinary albumin excretion and microalbuminuria in pre-diabetes subjects. Zhonghua Nei Ke Za Zhi 43: 170-173, 2004 (In Chinese). 
30. Stirban A, Gawlowski T and Roden M: Vascular effects of advanced glycation endproducts: Clinical effects and molecular mechanisms. Mol Metab 3: 94-108, 2013.

31. Sakai H, Jinde K, Suzuki D, Yagame M and Nomoto Y: Localization of glycated proteins in the glomeruli of patients with diabetic nephropathy. Nephrol Dial Transplant 11 (Suppl 5): S66-S71, 1996

32. Rösen P, Nawroth PP, King G, Möller W, Tritschler HJ and Packer L: The role of oxidative stress in the onset and progression of diabetes and its complications: A summary of a Congress Series sponsored by UNESCO-MCBN, the American diabetes association and the german diabetes society. Diabetes Metab Res Rev 17: 189-212, 2001.

33. Iwasaki Y, Sawada T, Kijima H, Kosuge T, Katoh M, Rokkaku K, Kita J, Shimoda M and Kubota K: Estimated glomerular filtration rate is superior to measured creatinine clearance for predicting postoperative renal dysfunction in patients undergoing pancreatoduodenectomy. Pancreas 39: 20-25, 2010.

34. Yan LJ: Analysis of oxidative modification of proteins. Curr Protoc Protein Sci Chapter 14: Unit14.4, 2009.

35. Booth AA, Khalifah RG, Todd P and Hudson BG: In vitro kinetic studies of formation of antigenic advanced glycation end products (AGEs). Novel inhibition of post-Amadori glycation pathways. J Biol Chem 272: 5430-5437, 1997.

36. Miyoshi H, Taguchi T, Sugiura M, Takeuchi M, Yanagisawa $K$, Watanabe Y, Miwa I, Makita Z and Koike T: Aminoguanidine pyridoxal adduct is superior to aminoguanidine for preventing diabetic nephropathy in mice. Horm Metab Res 34: 371-377, 2002.

37. Wagener FA, Dekker D, Berden JH, Scharstuhl A and van der Vlag J: The role of reactive oxygen species in apoptosis of the diabetic kidney. Apoptosis 14: 1451-1458, 2009.

38. Singh DK, Winocour P and Farrington K: Oxidative stress in early diabetic nephropathy: Fueling the fire. Nat Rev Endocrinol 7: 176-184, 2011.

39. Krishan $P$ and Chakkarwar VA: Diabetic nephropathy: Aggressive involvement of oxidative stress. J Pharm Educ Res 2: 35, 2011.

40. Mima A: Inflammation and oxidative stress in diabetic nephropathy: New insights on its inhibition as new therapeutic targets. J Diabetes Res 2013: 248563, 2013.

41. Shao N, Kuang HY, Wang N, Gao XY, Hao M, Zou W and Yin HQ: Relationship between oxidant/antioxidant markers and severity of microalbuminuria in the early stage of Nephropathy in type 2 diabetic Patients. J Diabetes Res 2013: 232404, 2013.

42. Grover HS and Luthra S: Molecular mechanisms involved in the bidirectional relationship between diabetes mellitus and periodontal disease. J Indian Soc Periodontol 17: 292-301, 2013.
43. Iacopino AM: Periodontitis and diabetes interrelationships: Role of inflammation. Ann Periodontol 6: 125-137, 2001.

44. Howard BV: Lipoprotein metabolism in diabetes mellitus. J Lipid Res 28: 613-628, 1987.

45. Ma Y, Wang Y, Huang Q, Ren Q, Chen S, Zhang A, Zhao L, Zhen $\mathrm{Q}$ and Peng Y: Impaired $\beta$ cell function in Chinese newly diagnosed type 2 diabetes mellitus with hyperlipidemia. J Diabetes Res 2014: 493039, 2014.

46. Moorhead JF, Chan MK, El-Nahas M and Varghese Z: Lipid nephrotoxicity in chronic progressive glomerular and tubulo-interstitial disease. Lancet 2: 1309-1311, 1982.

47. Krolewski AS, Warram JH and Christlieb AR: Hypercholesterolemia-a determinant of renal function loss and deaths in IDDM patients with nephropathy. Kidney Int Suppl 45: S125-S131, 1994.

48. Ravid M, Neumann L and Lishner M: Plasma lipids and the progression of nephropathy in diabetes mellitus type II: Effect of ACE inhibitors. Kidney Int 47: 907-910, 1995.

49. Rosario RF and Prabhakar S: Lipids and diabetic nephropathy. Curr Diab Rep 6: 455-462, 2006.

50. Lee HB, Yu MR, Yang Y, Jiang Z and Ha H: Reactive oxygen species-regulated signaling pathways in diabetic nephropathy. J Am Soc Nephrol 14 (8 Suppl 3): S241-S245, 2003.

51. Choudhary $\mathrm{N}$ and Ahlawat RS: Interleukin- 6 and C-reactive protein in pathogenesis of diabetic nephropathy: New evidence linking inflammation, glycemic control and microalbuminuria. Iran J Kidney Dis 2: 72-79, 2008.

52. Branchetti E, Poggio P, Sainger R, Shang E, Grau JB, Jackson BM, Lai EK, Parmacek MS, Gorman RC, Gorman JH, et al: Oxidative stress modulates vascular smooth muscle cell phenotype via CTGF in thoracic aortic aneurysm. Cardiovasc Res 100: 316-324, 2013.

53. Dalla Vestra M, Mussap M, Gallina P, Bruseghin M, Cernigoi AM, Saller A, Plebani M and Fioretto P: Acute-phase markers of inflammation and glomerular structure in patients with type 2 diabetes. J Am Soc Nephrol 16 (Suppl 1): S78-S82, 2005.

54. Sekizuka K, Tomino Y, Sei C, Kurusu A, Tashiro K, Yamaguchi Y, Kodera S, Hishiki T, Shirato I and Koide H: Detection of serum IL-6 in patients with diabetic nephropathy. Nephron 68: 284-285, 1994.

55. Chen FQ, Wang J, Liu XB, Ma XY, Zhang XB, Huang T, Ma DW and Wang QY: Levels of inflammatory cytokines in type 2 diabetes patients with different urinary albumin excretion rates and their correlation with clinical variables. J Diabetes Res 2013: 138969, 2013. 